Stand und Perspektiven des ökologischen Landbaus in Polen

\title{
Anstoß durch EU-Beitritt?
}

\section{Die politisch angestrebte Modernisierung der polnischen Landwirtschaft ist auf die Integration in die Europäische Union ausgerichtet. Ökologischer Landbau spielt dabei trołz seiner Potenziale nur eine geringe Rolle. Für eine stärkere Verbreitung müssen noch zahlreiche Hemmnisse überwunden werden.}

$\mathrm{N}$ Von Anke Poprawa ach Auflösung der sozialistischen Großbetriebe konnten in Polen neue landwirtschaftliche Betriebsstrukturen geschaffen werden. Einige landwirtschaftliche Betriebe stellten sich auf eine ökologische Wirtschaftsweise um. Ökologischer Landbau bietet Beschäftigungs- und auf die Region ausgerichtete Potenziale. Daher dürfte er prinzipiell gerade in Polen, wo noch fast ein Viertel der Beschäftigten in der Landwirtschaft tätig ist, eine gute Basis für einen ökologischen Strukturwandel bieten. Bis jetzt ist die ökologisch bewirtschaftete Fläche aber mit 0,3 Prozent nur $\mathrm{zu}$ einem geringen Anteil an der allgemeinen landwirtschaftlichen Produktion vertreten. In Deutschland liegt dieser Anteil bei 2,68 Prozent und in der EU bei 2,89 Prozent (1).

Ein Grund für diese schwache Entwicklung des ökologischen Landbaus in Polen war die einseitige Orientierung der polnischen Regierung auf eine maximale Agrarproduktion. Erst seit 2000 befasst sich die Regierung mit der Schaffung von gesetzlichen Rahmenrichtlinien und Förderprogrammen. Damit ist der Grundstein für einen Zuwachs der ökologisch produzierenden Betriebe und eine EU-weite Anerkennung der Vermarktung ökologisch produzierter Waren gelegt.

Für den Export von Ökowaren müssen die Landwirte nach den Richtlinien der EG-Verordnung zum ökologischen Landbau von 1991 produzieren. Mit dieser Verordnung wurde für den Schutz der Verbraucher vor Täuschung und der Wirtschaftsbeteiligten vor unlauterem Wettbewerb bei Erzeugnissen aus ökologischem Landbau ein einheitlicher Rechtsrahmen innerhalb der EU geschaffen. Mit Inkrafttreten dieser Verordnung dürfen in keinem EU-Land Produkte als „ökoprodukte" vermarktet werden, die nicht gemäß der Verordnung erzeugt wurden. Ökoimportware aus Drittländern kann nur dann in der EU als ökologisch vermarktet werden, wenn das Herkunftsland in einer von der EU-Kommission erstellten Liste aufgeführt ist. Hierfür müssen die
Länder nachweisen, dass sie in der Lage sind, ihre ökologischen Produkte gemäß einem dem EU-Standard gleichwertigen Verfahren zu erzeugen und zu kontrollieren (2).

Polen hat diesen Status noch nicht; polnische Ökoprodukte müssen für eine EU-weite Vermarktung daher noch komplizierte Genehmigungsverfahren durchlaufen. Der polnische Verband für ökologischen Landbau EKOLAND ist bemüht diesen Zustand zu ändern. Sein Kontrollverein Agro Bio Test e.V. will die amtliche Akkreditierung als polnische Kontrollstelle für den ökologischen Landbau gemäß der Norm EN-45011 beantragen.

Bis 1996 hat EKOLAND eine steigende Mitgliederzahl und Flächenzunahme registriert. 1997 und 1998 sind die Mitgliedszahlen hingegen gesunken und erst jüngst wieder angestiegen (vgl. Tabelle 1). Der Rückgang ist auf schlechte Positionen am Markt, fehlende Marktorientierung, das Fehlen staatlicher Unterstïtzung und die zusätzliche Kostenbelastung durch das Kontroll- und Zertifikatsprogramm zurückzuführen.

Größere Schwierigkeiten haben die polnischen Landwirte mit den Zoll- und Steuergebühren, den Registrier- und Analysekosten sowie mit der EUBioverordnung. Auf Grund dessen wird der Export in die EU immer noch in bescheidenem Rahmen betrieben und beläuft sich derzeit auf nur wenige Lastwagen pro Jahr. Vor allem schwarze Johannisbeeren, Erdbeeren, Zwiebeln und getrocknetes Gemüse werden vorwiegend in die Niederlande und nach Deutschland geliefert.

\begin{tabular}{|lcc|}
\hline \multicolumn{2}{|c|}{ Tabelle: } & Mitgliedsbetriebe bei EKOLAND \\
\hline Jahr & Betriebe & Fläche (ha) \\
\hline 1990 & 27 & 300 \\
\hline 1992 & 94 & 1.240 \\
1994 & 225 & 3.540 \\
1996 & 236 & 6.855 \\
1997 & 207 & 6.000 \\
1998 & 182 & 5.546 \\
1999 & 243 & 5.578 \\
\hline
\end{tabular}

Ökologischer Landbau in Osteuropa. ZMP, Bonn 1998.
Das Landwirtschaftsministerium zeigt nach langem Zögern endlich Interesse und versucht die ökologische Landwirtschaft finanziell zu fördern. Seit 1993 führt das Ministerium Boden- und Wasseranalysen durch. Die Arbeitsgemeinschaft für Bio-Anbau unterbreitete 1998 dem Landwirtschaftsminister Vorschläge für den Einsatz der Subventionen im Bio-Anbau. Daraufhin investierte der Staat 1998 in einige Untersuchungen zum biologischen Anbau. 1998 wurden bescheidene Subventionen für Kontroll- und Inspektionskosten zugewiesen.

Polen steht daher in den nächsten Jahren im ökologischen Landbau vor großen Herausforderungen (3). Diese liegen vor allem in den Bereichen:

- Schaffung gesetzlicher Rahmenrichtlinien,

- Optimierung der staatlichen Förderungsmaßnahmen und

- Verbesserung des Qualitäts- und Vermarktungsmanagements.

Einige Hindernisse, wie die in geringem Maße betriebene Weiterverarbeitung, unzureichende Aufklärung der KonsumentInnen und das Fehlen des ökologischen Landbaus als Forschungsgebiet an den entsprechenden Institutionen müssen noch weitestgehend beseitigt werden, damit im Endeffekt dem EU-Beitritt und der damit verbundenen europäischen Markteroberung nichts mehr im Wege steht. Dies geht aber nur wenn eine intensive Zusammenarbeit der polnischen Regierung mit den Institutionen des ökologischen Landbaus und den Landwirten stattfindet.

\section{Anmerkungen}

(1) Angaben der Stiffung Ökologie \& Landbau, www.soel. de

(2) Europäische Kommission (Hrsg.): Der ökologische Landbau - Ein Leiffaden zur EU-Gesetzgebung, Amt für amtliche Veröffentlichungen der $\mathrm{EG}$, Brüssel 2000.

(3) Vgl. dazu ausführlich Poprawa, A.: Stand und Perspektiven des ökologischen Landbaus in Polen. Unveröffentlichte Diplomarbeit, Rostock 2001. 
(c) 20I0 Authors; licensee IÖW and oekom verlag. This is an article distributed under the terms of the Creative Commons Attribution Non-Commercial No Derivates License (http://creativecommons.org/licenses/by-nc-nd/3.o/), which permits unrestricted use, distribution, and reproduction in any medium, provided the original work is properly cited. 